\title{
Cavernous Sinus Cavernous Hemangioma Largely Extending Into the Sella Turcica and Mimicking Pituitary Adenoma
}

\author{
-Case Report-
}

\author{
Satoshi HORI, Nakamasa HAYASHI, Kazuhiro NOMOTO*, Hikari SATO, \\ Tomohide HAYASHI, Shoichi NAGAI, Manabu NiSHIKATA**, and Shunro ENDO
}

Department of Neurosurgery, Faculty of Medicine, University of Toyama, Toyama;

* Laboratory of Pathology, Toyama University Hospital, Toyama;

${ }^{* *}$ Toyama Cyber Knife Center, Toyama

\begin{abstract}
A 77-year-old female presented with a rare cavernous sinus cavernous hemangioma with extension to the sella turcica, neuroradiologically mimicking nonfunctioning pituitary macroadenoma. The lesion was partially removed via transsphenoidal surgery, and the histological diagnosis was cavernous hemangioma. After stereotactic radiosurgery using a cyber knife, the lesion decreased in size. Stereotactic radiosurgery may be a good option for cavernous sinus cavernous hemangioma with high risk of surgical bleeding.
\end{abstract}

Key words: cavernous sinus cavernous hemangioma, magnetic resonance imaging, pituitary macroadenoma, stereotactic radiosurgery, transsphenoidal surgery

\section{Introduction}

Cavernous hemangiomas are benign vascular malformations which may originate in any intracranial region, account for 5 to $13 \%$ of intracranial vascular malformations, and occur in approximately 0.5 to $1 \%$ of the population. ${ }^{4,13)}$ The most common location is the cerebral hemispheres and the typical manifestations are seizures and bleeding. ${ }^{1,18)}$ Cavernous sinus cavernous hemangiomas are extremely rare and account for less than $2 \%$ of all cavernous sinus tumors. ${ }^{5,8)}$ We treated an elderly patient with an enhanced lesion in the cavernous sinus with extension to the sella turcica under a neuroradiological diagnosis of nonfunctioning pituitary adenoma, but the histological diagnosis was cavernous hemangioma. We discuss the difficulty of preoperative radiological distinction between cavernous hemangioma largely extending into sella turcica and pituitary macroadenoma, and a useful option for treatment of the surgically high-risk cavernous sinus cavernous hemangioma.

\section{Case Report}

A 77-year-old female suffered head trauma. Computed tomography revealed a high-density mass in the sellar and left parasellar regions. Magnetic resonance (MR) imaging showed a large, lobulated lesion in the sella turcica and

Received June 22, 2009; Accepted August 12, 2009 left cavernous sinus, which extended into the suprasellar region and compressed the optic chiasm (Fig. 1A-C). The lesion appeared homogeneously isointense on $\mathrm{T}_{1}$-weighted images and markedly hyperintense on $\mathrm{T}_{2}$-weighted images. The pituitary stalk and pituitary gland were markedly shifted to the right (Fig. 1A, B). The mass was homogeneously enhanced after administration of contrast medium. The patient had no neurological deficits. Static perimeter showed no visual defect. Preoperative basal values of thyroid-stimulating hormone, growth hormone, luteinizing hormone, follicle-stimulating hormone, prolactin, cortisol, and adrenocorticotropic hormone were 5.42 $\mu \mathrm{IU} / \mathrm{ml}, 0.07 \mathrm{ng} / \mathrm{ml}, 9.2 \mathrm{mIU} / \mathrm{ml}, 26.7 \mathrm{mIU} / \mathrm{ml}, 16.6 \mathrm{ng} / \mathrm{ml}$, $11.8 \mu \mathrm{g} / \mathrm{dl}$, and $46 \mathrm{pg} / \mathrm{ml}$, respectively. The preoperative diagnosis was nonfunctioning pituitary macroadenoma.

Surgical exploration via a sublabial transsphenoidal approach to the sellar lesion revealed a reddish fibrous hard tumor. The tumor was vascular, and was removed piecemeal with bipolar coagulation. Intraoperative examination of frozen sections established that the lesion was a cavernous hemangioma. The tumor was partially removed to obtain specimens for histological diagnosis.

The postoperative course was uneventful. Histological examination showed the tumor consisted mainly of multiple dilated thin-walled vascular channels (Fig. 2). The histological diagnosis was cavernous hemangioma. Cyber knife treatment (marginal dose $17 \mathrm{~Gy}$ and central dose 21 Gy/3 fractions/week) was given. The tumor had decreased in size at 1 year after radiosurgery (Fig. 1D). 

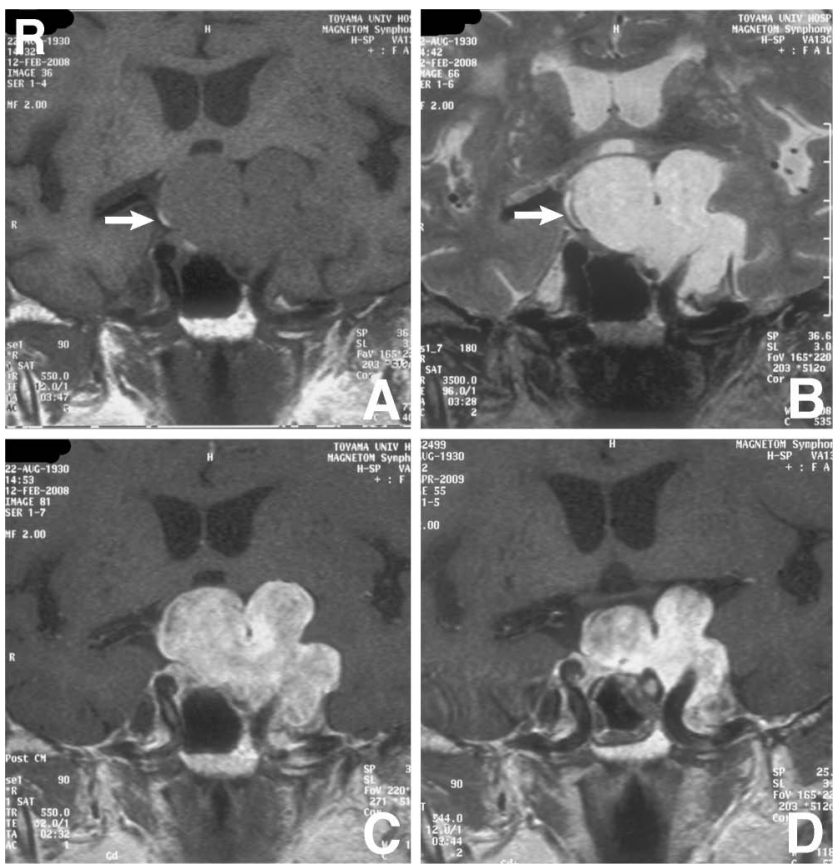

Fig. 1 A-C: Preoperative coronal $T_{1}$-weighted magnetic resonance (MR) image (A) showing a large, lobulated isointense lesion in the sella turcica and left cavernous sinus. Preoperative coronal $T_{2}$-weighted $M R$ image (B) showing a markedly hyperintense lesion. Preoperative coronal $T_{1}$-weighted MR image with contrast medium (C) showing homogeneous enhancement of the lesion. Arrows indicate the pituitary gland. D: Coronal $T_{1}$-weighted MR image with contrast medium 1 year after radiosurgery showing decreased size of the lesion.

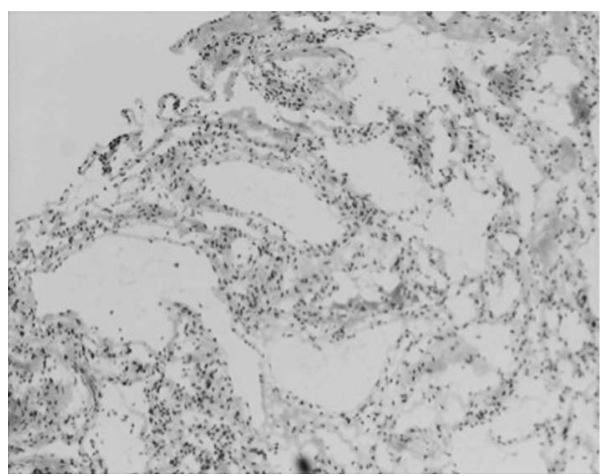

Fig. 2 Photomicrograph of the surgical specimen showing multiple dilated vascular channels with thin walls. Hematoxylin and eosin stain, $\times 400$.

\section{Discussion}

Extra-axial cavernous hemangiomas are rare vascular tumors, accounting for only 0.4 to $2 \%$ of the intracranial cavernous hemangiomas, and are most commonly located in the cavernous sinus or middle cranial fossa. ${ }^{22)}$ The clinical signs of cavernous sinus cavernous hemangioma are headache, ocular pain, and dysfunctions of the cranial nerves passing through the cavernous sinus, especially ptosis and diplopia. Sudden neurological deterioration in association with hemorrhage is rare..$^{9,20,22)}$

Cavernous sinus cavernous hemangiomas tend to grow as asymmetrical dumbbell-shaped masses occupying the middle cranial fossa and sellar lesion. ${ }^{20}$ Cavernous hemangiomas are difficult to differentiate from other cavernous sinus tumors like meningioma, neurinoma, or lateral extensions of pituitary adenoma. The preoperative misdiagnosis rate is reported as up to 66.7 to $87.5 \% .^{9,14,22)}$ In the present case, our preoperative diagnosis was nonfunctioning pituitary macroadenoma. MR imaging demonstrates pituitary adenomas are hypointense to isointense on $\mathrm{T}_{1}$-weighted images and variable intensity on $\mathrm{T}_{2^{-}}$ weighted images. Cavernous hemangioma may resemble pituitary adenoma, apart from the prominent hyperintensity on $\mathrm{T}_{2}$-weighted images. . $^{9,12,17,20)}$

Cavernous sinus cavernous hemangiomas with sellar or intracranial extension have three patterns of parasellar growth: endophytic lateral growth, endophytic medial growth, and exophytic growth. ${ }^{12)}$ In the endophytic medial growth pattern, lesions extend into the sella turcica, and can be differentiated from intrasellar cavernous hemangioma with cavernous sinus extension based on location of the major portion of the tumor. ${ }^{2-4,15,19)}$ In the present case, MR imaging showed that the pituitary gland was compressed to the lower right side in the sella turcica. Therefore, we speculate that the main mass of the cavernous hemangioma was located in the left cavernous sinus, and then expanded into the sella turcica.

Cavernous hemangioma is a highly vascular lesion. Despite recent advances in microsurgery, surgical removal of cavernous sinus cavernous hemangioma is often very difficult, because of the critical anatomical structures in the cavernous sinus and the risk of excessive intraoperative bleeding. $9,16,17,20,21)$ If cavernous hemangioma is suspected, frozen sections should be examined for diagnosis with partial resection after adequate preparation, since complete excision may result in serious bleeding. ${ }^{3,4)}$

Adjunctive treatment of residual cavernous hemangioma with stereotactic radiosurgery may yield excellent response and avoidance of morbidity ${ }^{6,8,10,11)}$ Radiosurgery can effectively reduce the rebleeding rate after the first symptomatic hemorrhage and may be useful in reducing the severity of seizures in patients with cavernous hemangioma. ${ }^{11)}$ Various treatment approaches toward cavernous sinus cavernous hemangioma have been reported. ${ }^{5}$ Radiosurgery is effective for cavernous sinus cavernous hemangioma. ${ }^{7-9,16,17,20)}$ Partial resection followed by stereotactic radiosurgery may be a useful option for the treatment of cavernous sinus cavernous hemangioma in elderly patients.

\section{References}

1) Acciarri N, Padovani R, Giulioni M, Gaist G, Acciarri R: Intracranial and orbital cavernous angiomas: a review of 74 surgical cases. Br J Neurosurg 7: 529-539, 1993

2) Buonaguidi R, Canapicci R, Mimassi N, Ferdeghini M: In- 
trasellar cavernous hemangioma. Neurosurgery 14: 732-734, 1984

3) Chuang CC, Jung SM, Yang JT, Chang CN, Pai PC: Intrasellar cavernous hemangioma. J Clin Neurosci 13: 672-675, 2006

4) Cobbs CS, Wilson CB: Intrasellar cavernous hemangioma: case report. J Neurosurg 94: 520-522, 2001

5) Fraser JF, Mass AY, Brown S, Anand VK, Schwartz TH: Transnasal endoscopic resection of a cavernous sinus hemangioma: technical note and review of the literature. Skull Base 18: 309-315, 2008

6) Hasegawa T, McInerney J, Kondziolka D, Lee JYK, Flickinger JC, Lunsford LD: Long-term results after stereotactic radiosurgery for patients with cavernous malformations. Neurosurgery 50: 1190-1198, 2002

7) Ivanov $P$, Chernov $M$, Hayashi M, Nakaya K, Izawa M, Murata N, Kubo O, Ujiie H, Muragaki Y, Nakamura R, Iseki H, Hori T, Takakura K: Low-dose gamma knife radiosurgery for cavernous sinus hemangioma: report of 3 cases and literature review. Minim Invasive Neurosurg 51: 140-146, 2008

8) Iwai Y, Yamanaka K, Nakajima H, Yasui T: Stereotactic radiosurgery for cavernous sinus cavernous hemangioma: case report. Neurol Med Chir (Tokyo) 39: 288-290, 1999

9) Kida Y, Kobayashi T, Mori Y: Radiosurgery of cavernous hemangioma in the cavernous sinus. Surg Neurol 56: 117-123, 2001

10) Kim DG, Choe WJ, Paek SH, Chung HT, Kim IH, Han DH: Radiosurgery of intracranial cavernous malformations. Acta Neurochir (Wien) 144: 869-878, 2002

11) Liu KD, Chung WY, Wu HM, Shiau CY, Wang LW, Guo WY, Pan DHC: Gamma knife surgery for cavernous hemangiomas: an analysis of 125 patients. J Neurosurg 102: 81-86, 2005

12) Lombardi D, Giovaneli M, Tribolet N: Sellar and parasellar extra-axial cavernous hemangiomas. Acta Neurochir (Wien) 130: $47-54,1994$
13) Maraire JN, Awad IA: Intracranial cavernous malformations: lesion behavior and management strategies. Neurosurgery 37: 591-605, 1995

14) Mendonca JLF, Viana SL, Matsumine M, Silva RF, Viana MA, Freitas FM: Cavernous angioma of the cavernous sinus: imaging findings. Arq Neuropsiquiatr 62: 1004-1007, 2004

15) Mitsuhashi $T$, Hashimoto R, Nagahama $S$, Nagata $Y$ : Intrasellar cavernous hemangioma in neurofibromatosis. Hum Pathol 22: 623-624, 1991

16) Nakamura $N$, Shin $M$, Tago $M$, Terahara A, Kurita $H$, Nakagawa K, Ohtomo K: Gamma knife radiosurgery for cavernous hemangiomas in the cavernous sinus. J Neurosurg 97: 477-480, 2002

17) Peker S, Kilic T, Sengoz M, Pamir MN: Radiosurgical treatment of cavernous sinus cavernous hemangiomas. Acta Neurochir (Wien) 146: 337-341, 2004

18) Robinson JR, Awad IA, Litle JR: Natural history of the cavernous angioma. J Neurosurg 75: 709-714, 1991

19) Sansone ME, Liwnicz BH, Mandybur TI: Giant pituitary cavernous hemangioma: case report. J Neurosurg 53: 124-126, 1980

20) Shi J, Hang C, Pan Y, Liu C, Zhang Z: Cavernous hemangiomas in the cavernous sinus. Neurosurgery 45: 1308-1314, 1999

21) Shi J, Wang H, Hang C, Pan Y, Liu C, Zhang Z: Cavernous hemangiomas in the cavernous sinus. Case report. Surg Neurol 52: 473-479, 1999

22) Zhou LF, Mao Y, Chen L: Diagnosis and surgical treatment of cavernous sinus hemangiomas: an experience of 20 cases. Surg Neurol 60: 31-38, 2003

Address reprint requests to: Nakamasa Hayashi, M.D., Department of Neurosurgery, Faculty of Medicine, University of Toyama, 2630 Sugitani, Toyama 930-0194, Japan. e-mail:nakamasa@iwa.att.ne.jp 